\title{
Characterization of More Selective Central Nervous System Nrf2-Activating Novel Vinyl Sulfoximine Compounds Compared to Dimethyl Fumarate
}

\author{
Karl E. Carlström ${ }^{1}$ • Praveen K. Chinthakindi ${ }^{2,3}$ • Belén Espinosa ${ }^{4}$ • Faiez Al Nimer ${ }^{1}$ • Elias S. J. Arnér ${ }^{4}$. \\ Per I. Arvidsson ${ }^{2,5}$ • Fredrik Piehl $^{1} \cdot$ Katarina Johansson $^{4,6}$
}

Published online: 11 May 2020

(C) The Author(s) 2020

\begin{abstract}
The Nrf2 transcription factor is a key regulator of redox reactions and considered the main target for the multiple sclerosis (MS) drug dimethyl fumarate (DMF). However, exploration of additional Nrf2-activating compounds is motivated, since DMF displays significant off-target effects and has a relatively poor penetrance to the central nervous system (CNS). We de novo synthesized eight vinyl sulfone and sulfoximine compounds (CH-1-CH-8) and evaluated their capacity to activate the transcription factors Nrf2, NF KB, and HIF1 in comparison with DMF using the pTRAF platform. The novel sulfoximine CH-3 was the most promising candidate and selected for further comparison in vivo and later an experimental model for traumatic brain injury (TBI). CH-3 and DMF displayed comparable capacity to activate Nrf2 and downstream transcripts in vitro, but with less offtarget effects on HIF 1 from CH-3. This was verified in cultured microglia and oligodendrocytes (OLs) and subsequently in vivo in rats. Following TBI, DMF lowered the number of leukocytes in blood and also decreased axonal degeneration. CH-3 preserved or increased the number of pre-myelinating OL. While both CH-3 and DMF activated Nrf2, CH-3 showed less off-target effects and displayed more selective OL associated effects. Further studies with Nrf2-acting compounds are promising candidates to explore potential myelin protective or regenerative effects in demyelinating disorders.
\end{abstract}

Key Words $\mathrm{Nrf2} \cdot \mathrm{NFKB} \cdot \mathrm{HIF} \cdot$ dimethyl fumarate $\cdot$ multiple sclerosis $\cdot$ redox regulation $\cdot$ pTRAF $\cdot$ sulfoximine $\cdot$ traumatic brain injury $\cdot$ microglia

Electronic supplementary material The online version of this article (https://doi.org/10.1007/s13311-020-00855-0) contains supplementary material, which is available to authorized users.

Karl E. Carlström

karl.carlstrom@ki.se

1 Department of Clinical Neurosciences, Section of Neurology, Karolinska Institutet, 17177 Stockholm, Sweden

2 Catalysis and Peptide Research Unit, University of KwaZulu Natal, Durban 4000, South Africa

3 Department of Medicinal Chemistry, Drug Design and Discovery, Uppsala University, Box 574, 75123 Uppsala, Sweden

4 Department of Medical Biochemistry and Biophysics, Division of Biochemistry, Karolinska Institutet, 17177 Stockholm, Sweden

5 Department of Medical Biochemistry and Biophysics, Science for Life Laboratory, Drug Discovery and Development Platform and Division of Translational Medicine and Chemical Biology, Karolinska Institutet, 17121 Solna, Sweden

6 Pfizer Innovation AB, 19190 Sollentuna, Sweden

$\begin{array}{ll}\text { Abbreviations } \\ \text { BBB } & \text { Blood-brain barrier } \\ \text { CSF } & \text { Cerebrospinal fluid } \\ \text { DMF } & \text { Dimethyl fumarate } \\ \text { HIF1 } & \text { Hypoxia-inducible factor 1 } \\ \text { Keap1 } & \text { Kelch-like ECH-associated protein 1 } \\ \text { MS } & \text { Multiple sclerosis } \\ \text { NFkB } & \text { Nuclear factor K-light-chain-enhancer } \\ & \text { of activated B cells } \\ \text { NFL } & \text { Neurofilament light } \\ \text { Nrf2 } & \text { Nuclear factor (erythroid-derived 2)-like 2 } \\ \text { PD } & \text { Parkinson's disease } \\ \text { pTRAF } & \text { Plasmid for transcription factor } \\ & \text { reporter activation based } \\ & \text { upon fluorescence } \\ \text { RRMS } & \text { Relapsing remitting multiple sclerosis } \\ \text { TBI } & \text { Traumatic brain injury } \\ \text { TNF } & \text { Tumor necrosis factor }\end{array}$

\section{Abbreviations}

CSF Cerebrospinal fluid

DMF Dimethyl fumarate

HIF1 Hypoxia-inducible factor 1

MS Multiple sclerosis

NFkB Nuclear factor K-light-chain-enhancer

NFL

Nrf2 Nuclear factor (erythroid-derived 2)-like 2

PD Parkinson's disease 


\section{Introduction}

The ability to manage oxidative and xenobiotic stress is a highly conserved process engaging several redox-regulated transcription factors [1], both during disease and in the healthy state [2]. Among these, nuclear factor (erythroid-derived 2)like 2 (Nrf2) is a well-characterized responder to oxidative stress via Kelch-like ECH-associated protein 1 (Keap1), and has been ascribed therapeutic relevance in various cell types during different pathologies [3-6]. In addition, Nrf2 has been suggested a potential therapeutic target during inflammatory conditions [7-9].

One striking example of successful clinical development of Nrf2-modulating drugs is use of dimethyl fumarate (DMF/Tecfidera ${ }^{\mathrm{TM}}$ ) which currently is a leading first-line treatment for relapsing remitting multiple sclerosis (RRMS) worldwide [10-12]. However, as DMF also engages additional transcription factors, it is unclear to what degree therapeutic effects can be associated with activation of solely Nrf2 [13-16]. As an example, apart from affecting Nrf2-regulated transcripts, DMF in RRMS patients also induces transcripts regulated by Nuclear factor $k$-light-chain-enhancer of activated $\mathrm{B}$ cells $(\mathrm{NF} \kappa \mathrm{B})$ and hypoxia-inducible factor (HIF) [17]. In addition, data on blood-brain barrier (BBB) penetrance by DMF, especially in human, is limited which may affect its therapeutic potential and motivates further research on other types of Nrf2 activators [18]. In line with this, there is a growing interest for novel Nrf2activating compounds and especially vinyl sulfone and sulfoximine compounds in the field of drug discovery [19-21].

The aim of this study was to synthesize and characterize more selective Nrf2-activating compounds able to target CNS-resident cells. Expression patterns were evaluated in vitro and in vivo following stimulation with DMF or one of eight synthesized compounds (CH-1-CH-8). The vinyl sulfoximine $\mathrm{CH}-3$ was selected for further in vivo studies in naïve rats and in an experimental model for traumatic brain injury (TBI), which is known to activate of $\mathrm{Nrf2}, \mathrm{NFkB}$, and HIF1 pathways, as well as displaying cell infiltration [16], focal immune cell activation [22], and neurodegeneration [4, 20].

\section{Methods}

\section{Transcriptional Response Analysis}

Assessing the transcriptional activities of $\mathrm{Nrf} 2, \mathrm{NF} \kappa \mathrm{B}$, and HIF1 was done using the pTRAF tool as described previously [14]. Generation of stable $\operatorname{HEK}\left(\mathrm{pTRAF}^{\mathrm{Nrf2} /}\right.$ $\mathrm{HIF} / \mathrm{NFkB}$ ) reporter cell line were described previously
(Sthijns MMJPE et al, 2017). Stably transfected $\operatorname{HEK}\left(\mathrm{pTRAF}^{\mathrm{Nrf2} / \mathrm{HIF} / \mathrm{NFkB}}\right.$ ) reporter cells were seeded in a collagen I-coated 96-well plates at a density of 18 000 cells/well and incubated overnight, followed by exposure of different transcription factor inducers as indicated (Thermo Fisher Scientific, Waltham, MA) [41]. Samples were loaded in as technical duplicates or triplicates. For microscopy, cell nuclei were stained with $40 \mathrm{ng} / \mathrm{ml}$ Hoechst for $30 \mathrm{~min}$ and subsequently fixated in $2 \%$ ice-cold paraformaldehyde for $10 \mathrm{~min}$ at RT. The Operetta High Content Imaging System with the Columbus System was used to analyze images and determine the mean fluorescence intensity per cell.

\section{In Vivo Experiments}

Dark Agouti (DA) rats were bred in the in-house breeding facility of the Karolinska University Hospital and fed standard rodent chow and water ad libitum. Animals were kept in open cages in a facility with 12 -h light/dark cycles. Animals were given vehicle CH-3 $(25 \mathrm{mg} / \mathrm{kg})$ or DMF $(25 \mathrm{mg} / \mathrm{kg})$ in $0.1 \%$ methyl cellulose via oral gavage in a total volume of $1 \mathrm{~mL}$ (Sigma, St. Louis, MO). For temporal assessments after single doses, groups of three animals were used for every time point. TBI was performed on 10-12-week-old DA males under deep isoflurane anesthesia using a weight-drop model [23]. Rats were placed in a stereotactic frame and injected with $250 \mu \mathrm{L}$ bupivacaine $2.5 \mathrm{mg} / \mathrm{mL}$ (Sigma, St. Louis, MO) under the skin. The skull bone was exposed and a small hole was drilled $3 \mathrm{~mm}$ posterior and $2.3 \mathrm{~mm}$ lateral of bregma. A focal cerebral contusion injury was made using a weight-drop device hitting a piston resting on the dura matter [24]. The piston was allowed to vertically dislocate the dura matter $3 \mathrm{~mm}$ in the vertical axis. Any local hemorrhage was stopped and the skin was closed using sutures. Animals were given $\mathrm{CH}-3$ $(25 \mathrm{mg} / \mathrm{kg})(n=7)$ or DMF $(25 \mathrm{mg} / \mathrm{kg})(n=7)$ or only vehicle $(n=6)$ as described in Fig. 4(D). All experiments were approved and performed in accordance with Swedish National Board of Laboratory Animals and the European Community Council Directive (86/609/EEC) under the permits N275-15 and N244-13.

\section{Flow Cytometric Analysis}

Rats were perfused with $100 \mathrm{~mL}$ PBS, and the brain was removed and minced using a sterile scalpel. The tissue was incubated in $1 \mathrm{~mL}$ Accutase and $4 \mu \mathrm{L}$ DNase $/ \mathrm{mL}$ at $+37{ }^{\circ} \mathrm{C}$ (Sigma, St. Louis, MO) and passed through a fire-polished Pasteur pipette every 10th minute, this was repeated three times with decreasing pipette opening. The homogenate was filtered through a 70- $\mu \mathrm{m}$ strainer and myelin was removed by a $37 \%$ Percoll (Sigma, St. Louis, MO) layer spun at $800 \times g$ for 10 min at $+10{ }^{\circ} \mathrm{C}$ without additional acceleration or brake. 
The pellet was re-suspended to a single cell suspension and stained for anti-O4, anti-Cd11b, and anti-Cd45 (Novus Biologicals, Littleton, CO). Dead cells were excluded using near IR Live/Dead probes (Thermo Fisher Scientific, Waltham, MA). Samples were analyzed with a 3-laser Beckman Coulter Gallios using Kaluza Software.

\section{Neurofilament Light Protein}

Rat cerebrospinal fluid (CSF), about $100 \mu \mathrm{L}$, was collected directly after sacrifice, prior to PBS perfusion, from the cerebellomedullary cistern and directly frozen and stored at $-70{ }^{\circ} \mathrm{C}$. Concentrations of neurofilament light protein $(\mathrm{NF})$ were determined using the NF-Light ELISA exactly according to the manufacturer's instructions (Umandiagnostics, Umeå, Sweden). Samples were diluted $1: 3$ and loaded in duplicates.

\section{Cell Cultures}

HEK293 cells and stable HEK(pTRAF ${ }^{\mathrm{Nrf2} / \mathrm{HIF} / \mathrm{NFkB}}$ ) reporter cells were cultured in Eagle's minimum essential medium, supplemented with $10 \%$ fetal bovine serum and $100 \mathrm{U}$ penicillin/ml and $100 \mu \mathrm{g}$ streptomycin $/ \mathrm{ml}$. Cells were cultured at $37{ }^{\circ} \mathrm{C}$ in a humidified atmosphere with $5 \% \mathrm{CO}_{2}$ and $21 \% \mathrm{O}_{2}$. Primary rat cultures were established from neonatal pups. Microglia and oligodendrocytes were isolated as described for flow cytometry; following Percoll layering, cells were labeled with either anti-cd11b or antiA2B5 Microbeads (Milteny Biotec, Bergisch, D). Microglia were cultured in DMEM/F12 supplemented with $100 \mathrm{U}$ penicillin/ml and $100 \mu \mathrm{g}$ streptomycin $/ \mathrm{ml}$ and $5 \%$ fetal bovine serum (Thermo Fisher Scientific, Waltham, MA). Oligodendrocytes were cultured in in $200 \mathrm{~mL}$ Neurobrew, 100 mL N2-supplement (Milteny Biotec, Bergisch, D), $2 \mathrm{~mL}$ bFGF (PeproTech, Princeton, NJ), and $2 \mathrm{~mL}$ PDGF-BB (R\&D Systems, Minneapolis, MA) per $10 \mathrm{~mL}$ of DMEM/F-12 media on poly-L-lysine precoated culture ware. For differentiation of oligodendrocytes, bFGF and PDGF-BB was removed $48 \mathrm{~h}$ prior to stimulation.

\section{Quantitative Real-Time PCR}

Total RNA was isolated from rat tissue or human or rat cells using RNeasy mini kit (Qiagen, Venlo, Netherlands) exactly as described by the manufacturer's instructions, including 15 min on-column DNase digestion. cDNA was prepared with reverse transcriptase PCR using iScript kit (BioRad Laboratories, Hercules, CA). Amplifications were conducted using Bio-Rad SYBR green according to the manufacturer's instructions and plates were run in BioRad CFX optical system, and samples were loaded in triplicates (BioRad Laboratories, Hercules, CA). Primers were designed to be optimal at $+60{ }^{\circ} \mathrm{C}$ and to span an exon-exon junction using online software at http://www. ncbi.nlm.nih.gov.

Human primers:

HPRT: F: CTCATGGACTGATTATGGACA;
R: GCAGGTCAGCAAAGAACTTAT
B-ACTIN: F: CATGTACGTTGCTATCCAGGC;
R: CTCCTTAATGTCACGCACGAT
GSTA4: F: TCAGCTGAGCCTTGCAGATGTGA;
R: GGGGGAGGCTTCTTCTTGCTGC
GCLM: F: CATTTACAGCCTTACTGGGAGG;
R: ATGCAGTCAAATCTGGTGGCA
TXN: F: ATATGGCAAGAAGGTGATGCTCC;
R: CGTGGCTGAGAAGTCAACTACTA
VEGF: F: TCTGCAGCTCTGTGTGAAGG;
R: ACTTCTCCACAACCCTCTGC
TXNDC17: F: GCAGGCTGAACCAGTCGTA;
R: TACCAGTTTTGAGGTGTTCCATA
iNOS: F: ACAAATTCAGGTACGCTGTG;
R: TGCACGAGCCTGTAGTG
IL8: F: TCTGCAGCTCTGTGTGAAGG;
R: ACTTCTCCACAACCCTCTGC
NQOI: F: AGTGCAGTGGTGTGATCTCG;
R: GGTGGATCACGCCTGTAAT
TXNRD1: F: ATATGGCAAGAAGGTGATGCTCC;
R: GGGCTTGTCCTAACAAAGCTG
HMOX1: F: CCGACAGCATGCCCCAGGATT;
R: GTCTCGGGTCACCTGGCCCTT

Rat Primers

Hprt: F: CTCATGGACTGATTATGGACA; R: GCAGGTCAGCAAAGAACTTAT B-actin: F: CGTGAAAAGATGACCCAGATCA; R: AGAGGCATACAGGGACAACACA Gclm: F: AGTGGGCACAGGTAAAACCC; R: ACTTGCCTCAGAGAGCAGTTC Nqo 1: F: CAGAAACGACATCACAGGGGA; R: GGCCTTCCTTATACGCCAGA Vegf: F: GGGAGCAGAAAGCCCATGAA; R: GCTGGCTTTGGTGAGGTTTG iNos: F: CAACATCAGGTCGGCCATTACT; R: TAGCCAGCGTACCGGATGA Il6: F: AGAAAAGAGTTGTGCAATGG; R: ACAAACTCCAGGTAGAAACG Txn: F: GTAGACGTGGATGACTGCCA; R: CTCCCCAACCTTTTGACCCTT Gsta4: F: CAGGAGTCATGGAAGTCAAAC; R: TTCTCATATTGTTCTCTCGTCTC 
Ark1b8: F: GACTTCCAGTTGAGCGACCA; R: TCCATGTTGACTGTCTCAGGC

Hmox 1: F: AGGGAAGGCTTTAAGCTGGT; R: AGGGAAGTAGAGTGGGGCAT

Bax: F: TTGCTACAGGGTTTCATCCAGG; R: CACTCGCTCAGCTTCTTGGT

\section{Statistical Analysis}

General statistical analyses were performed in GraphPad Prism software. Principal component analyses and hierarchy clustering were generated in $\mathrm{R}$ using ggbiplot. Comparisons between two groups were done with Student's two-tailed unpaired $t$ test. Comparisons between paired groups were done with paired $t$ test. Two group comparisons with a control group were done with one-way ANOVA with Benjamin-Hochberg post-hoc test. $P<0.05$ was throughout considered statistically significant.

\section{Results}

\section{Synthetization of the VSC2(CH-1) Analogues CH-2-CH-8}

Vinyl sulfone compounds, have been implicated as modulators of Nrf2 activity. Herein we first evaluated the vinyl sulfone compound VSC2 ((E)-1-(2-((2-methoxyphenyl)sulfonyl)vinyl)2-chlorobenzeneanalogues $/ \mathrm{CH}-1$, which has been suggested to activate Nrf2-regulated pathways [19-21] (Fig. 1(A, D), Supplementary Table 1). Exchange of the $-\mathrm{O}$ atom (i.e. sulfone $\mathrm{CH}-1$ ) with a $-\mathrm{NH}$ moiety yields a novel sulfoximine (CH-2) (Fig. 1(D)); such isosteric replacement may improve compound quality [25] as well as activity [26]. We thus synthetized sulfoximine compounds depicted in Fig. 1(D). Based on our earlier synthetic methodology reports, we have synthesized CH-2 [27] and carried out $N$-functionalization such as $\mathrm{N}$-methylation (CH-3) [28], $\mathrm{N}$-cyanation (CH-4) [29], $\mathrm{N}$ arylation (CH-7, 8) [30], and $\mathrm{N}$-acylation (CH-5, 6) (Fig. 1(D), Supplementary Table 1).

\section{CH-3 Is More Specific to Nrf2 Compared to DMF in PTRAF-Transfected HEK293 Cells}

DMF, which is commonly used in RRMS, mainly activates Nrf2, but is also suggested to have additional off-target effects. Thus, this is an incentive to carry out comparison to our newly synthesized compounds. For this, compounds (CH-1-CH-8) were initially evaluated in HEK293, stably transfected with plasmids for transcription factor reporter activation based upon fluorescence (pTRAF) [14] (Fig. 1(A)). The pTRAF approach enables simultaneous monitoring of DMF-affected transcription factors $\mathrm{Nrf} 2, \mathrm{NF} \mathrm{BB}$, and HIF1 at a single-cell level. $\mathrm{CH}-1, \mathrm{CH}-2$, and $\mathrm{CH}-3$ were selected for further
Fig. 1 Experimental outline and structures of newly synthetized compounds. (A) Initial screening of compound $\mathrm{CH}-1-8$ and $\mathrm{DMF}$ using pTRAF and qPCR. (B) Stimulation of primary glial cultures with DMF and $\mathrm{CH}-3$. (C) Evaluation of $\mathrm{CH}-3$ and $\mathrm{DMF}$ in an experimental model for traumatic brain injury. (D) Chemical structure of $\mathrm{CH}-1-8$ a

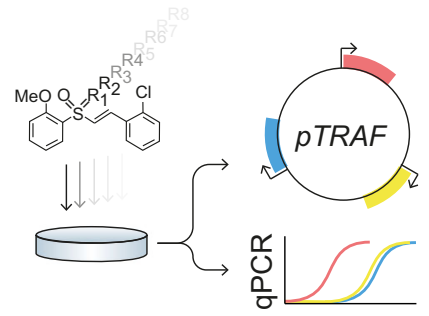

b

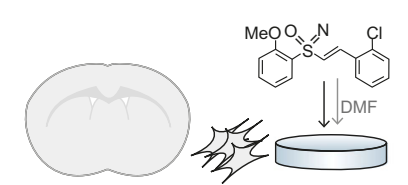

C

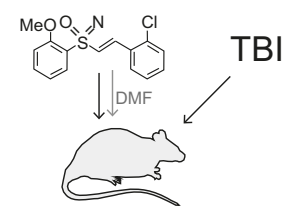

d<smiles>COc1ccccc1S(=O)(=O)/C=C/c1ccccc1Cl</smiles><smiles>COc1ccccc1S(=O)(=O)/C=C/c1ccccc1Cl</smiles>

$\mathrm{CH}-2$<smiles>COc1ccccc1S(=O)(=O)/C=C/c1ccccc1Cl</smiles><smiles>COc1ccccc1S(=O)(=O)N(C)C=Cc1ccccc1Cl</smiles><smiles>CCS(=O)(=NC(=O)C(F)(F)F)c1ccccc1OC</smiles><smiles>COc1ccc(/C=C/S(=O)(=O)c2ccccc2)cc1</smiles>

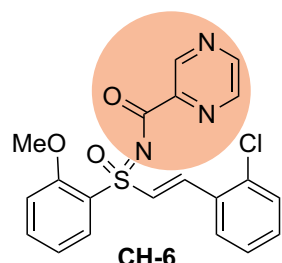
$\mathrm{CH}-6$<smiles>COc1ccccc1S(=O)(=NN(c1ccc(Cl)cc1)c1ccccc1Cl)c1ccccc1Cl</smiles> 
analysis based on their activity towards Nrf2 in combination with their cell viability profile (Fig. S1a, b). Optimal working concentrations were also determined for DMF, based on Nrf2activation and cell viability (Fig. S1c). CH-1, CH-2, and CH-3 all activated Nrf2 with limited off-target effects on NFKB and HIF1 (Fig. 2(A-D)). In contrast, while DMF activated Nrf2, it also activated HIF1 and downstream transcription of $V E G F$ (Fig. 2(B-E)). DMF also caused NF $k B$ activation, but without elevation of downstream $I L 8$ transcription (Fig. 2(D, E)). Both CH-3 and DMF showed increased Nrf2 activation upon costimulation with tumor necrosis factor (TNF) compared to
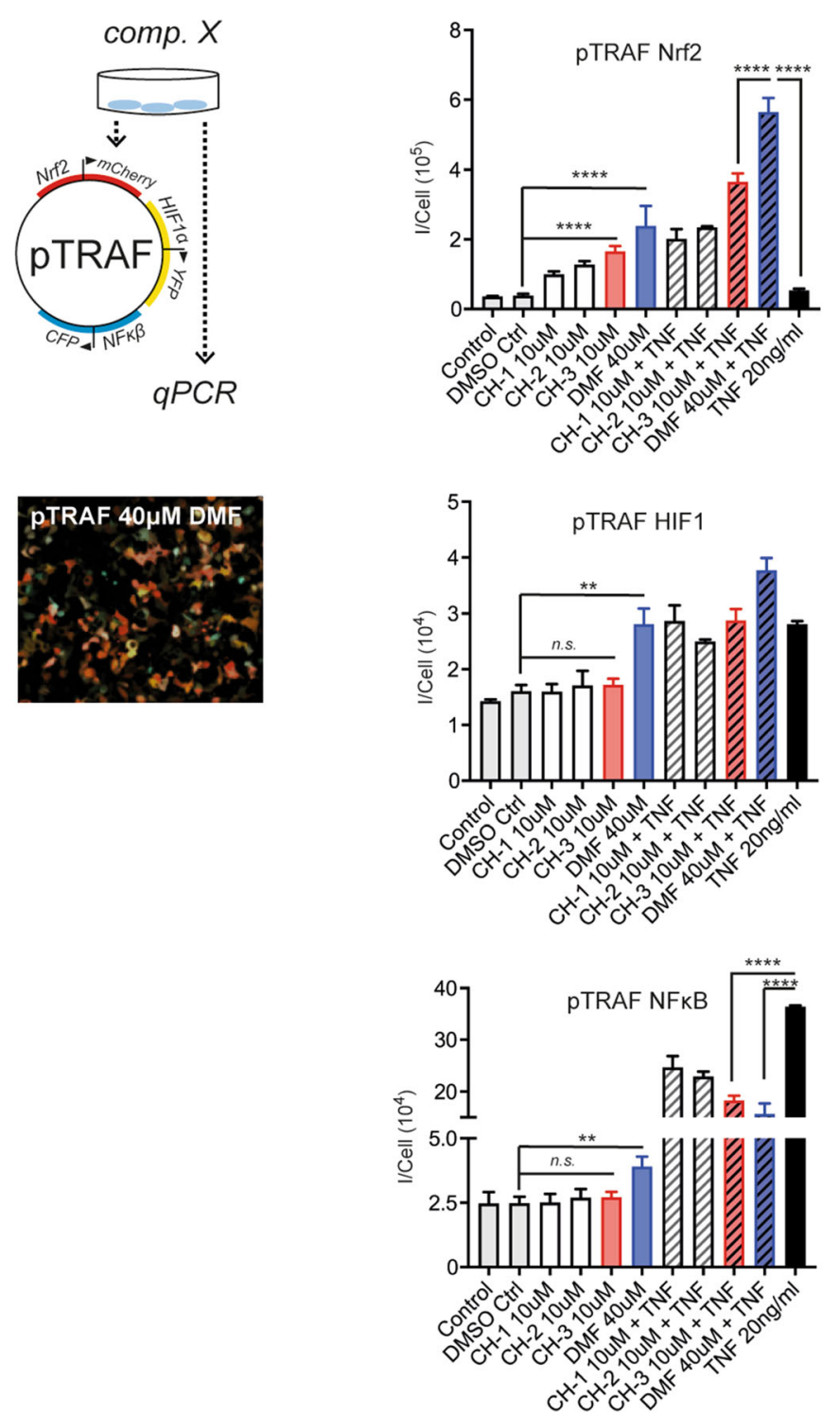

Fig. 2 Stable transfected HEK(pTRAF ${ }^{\mathrm{Nrf2} / \mathrm{HIF} / \mathrm{NFkB}}$ ) reporter cell line enables simultaneous detection of Nrf2, NFKB, and HIF1 activation. (A) Schematic illustration of analysis approach using pTRAF and qPCR. (B-D) Stimulation for $24 \mathrm{~h}$ with $10 \mu \mathrm{M}$ of $\mathrm{CH}-1$, $\mathrm{CH}-2$, and $\mathrm{CH}-3$ or $40 \mu \mathrm{M}$ DMF, with and without $20 \mathrm{ng} / \mathrm{mL} \mathrm{TNF}(n=$ only TNF (Fig. 2(B)). CH-3 was thus selected for further characterization and comparison to DMF (Fig. 1(A-C)).

To investigate downstream effects following transcriptional factor activation, we quantified ten transcripts known to be regulated by $\mathrm{Nrf} 2, \mathrm{NF} \kappa \mathrm{B}$, and/or HIF1. HEK(pTRAF ${ }^{\mathrm{Nrf2} / \mathrm{HIF} /}$ NFkB ) cells were stimulated with DMF $(40 \mu \mathrm{M})$ or $\mathrm{CH}-3$ (10 $\mu \mathrm{M}$ ) and among the evaluated transcripts, TXNRD1, TXNDC17 and VEGF appeared to differ between CH-3 and DMF stimulation (Fig. 2(E), Fig. S2). In contrast, transcripts generally associated with Nrf2, such as GSTA4, GCLM, HMOX1, and NQO1, did not differ in expression
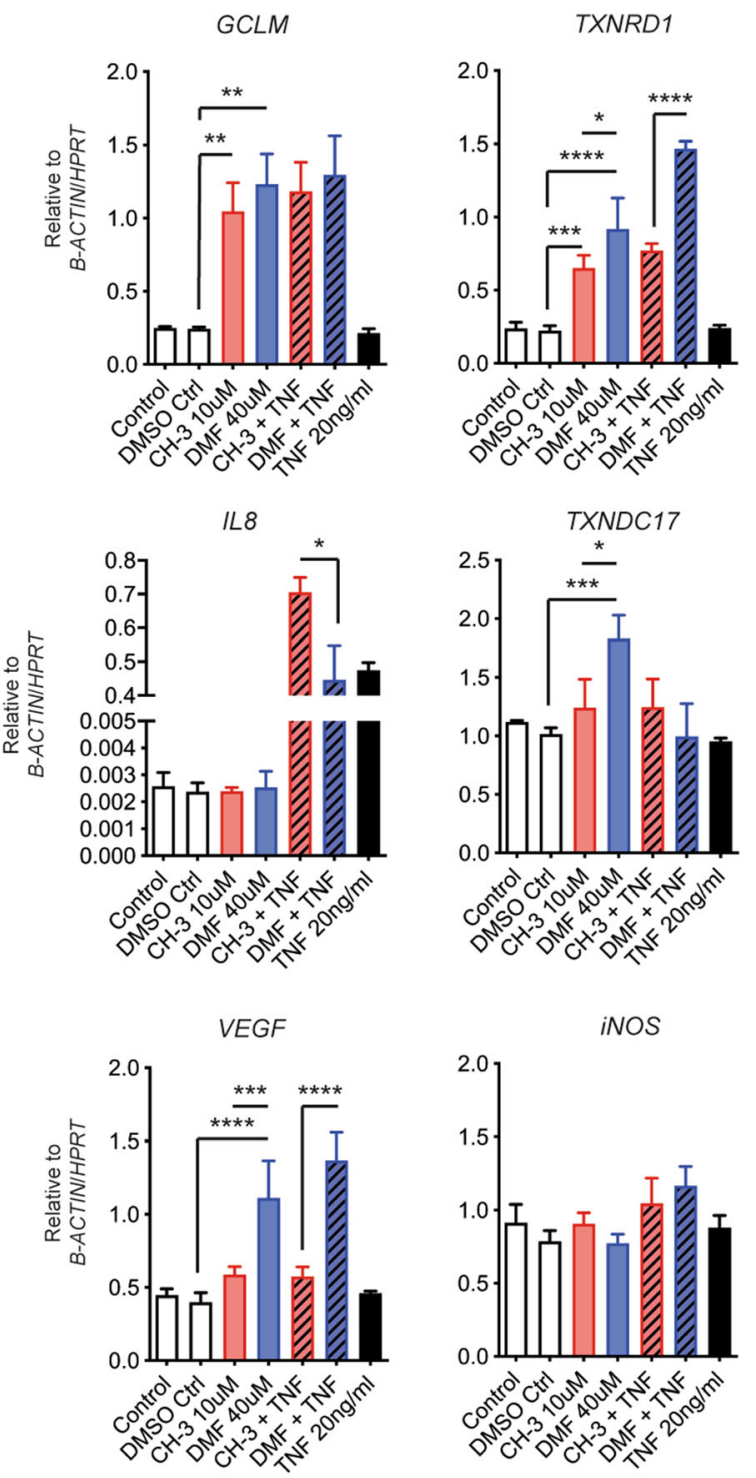

3). (E) Examples of transcription levels following $3 \mathrm{~h}$ of stimulations with DMF $(40 \mu \mathrm{M})$, CH-3 $(10 \mu \mathrm{M})$ with and without TNF $(20 \mathrm{ng} / \mathrm{mL})$. Errorbars show S.D. Two group comparisons with a control group were done with one-way ANOVA. $* P<0.05, * * P<0.01, * * * P<0.001$, $* * * * P<0.0001$ 


\section{in vitro stimulation with $\mathrm{CH}-3$ and $\mathrm{DMF}$}

HEK293 cells

a
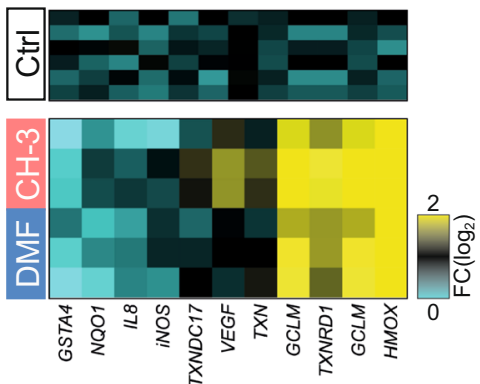

b

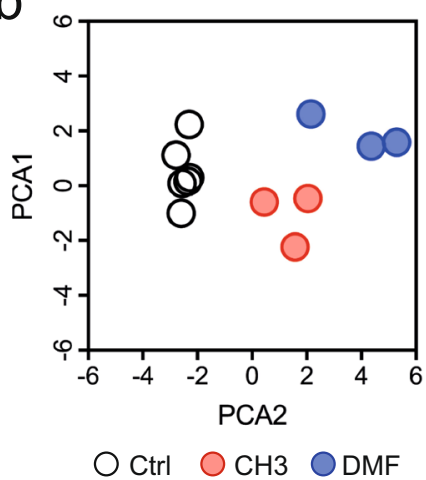

Fig. 3 Transcriptional profile in HEK293 cells and primary cultures of microglia and oligodendrocyte following DMF and CH-3. (A) Heat-map of transcript fold-change ( $\left.\log _{2}\right)$ following $\mathrm{CH}-3$ and DMF stimulation. (B) PCA reduction plot based on the transcription of genes indicated in the heat-map after $3 \mathrm{~h}$ of stimulations with $\mathrm{CH}-3$ (red), DMF (blue), or

between the two compounds (Fig. S2). This validated the results obtained from the pTRAF reporter system, i.e., that $\mathrm{CH}-3$ displayed a similar capacity as DMF to activate Nrf2 in vitro.

\section{Transcriptional Changes by CH-3 and DMF in Human Cells Are Partially Conserved in Rat Glial Cultures}

To illustrate the transcriptional profiles following $\mathrm{CH}-3$ or DMF stimulation, the expression of transcripts in Fig. 3(A) and Fig. S2 were reduced in a principal component analysis (PCA) plot. This indicated separate clustering of DMF compared to $\mathrm{CH}-3$, and also different from unstimulated samples (Fig. 3(B)). Further, Nrf2 is a suggested target in both microglia and oligodendrocytes (OLs) upon stimulation with vinyl sulfone or DMF, respectively $[12,20,31]$. In order to explore transcriptional patterns downstream of Nrf2, NFKB, and HIF1 and to verify HEK293 results in cell types relevant for the CNS, we established primary rat microglia and $\mathrm{OL}$ cultures and exposed them to $\mathrm{CH}-3$ or DMF
Primary rat glia

d

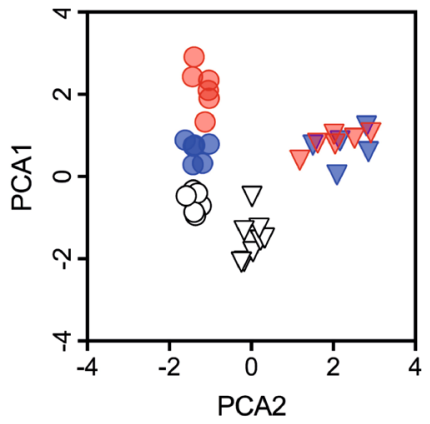

$\nabla$ Oligodendrocyte DMSO

$\checkmark$ Oligodendrocyte $\mathrm{CH}-3$

$\checkmark$ Oligodendrocyte DMF

Microglia DMSO

Microglia $\mathrm{CH}-3$

Microglia DMF unstimulated (white). (C) Heat-map of transcription levels following $3 \mathrm{~h}$ of stimulations with DMF $(15 \mu \mathrm{M}), \mathrm{CH}-3(10 \mu \mathrm{M})(n=6)$. (D) PCA reduction plot based on the transcription of genes indicated in the heatmap after $3 \mathrm{~h}$ of stimulations $(n=6)$

(Fig. S3a-d). The cultures were stimulated with either DMF $(15 \mu \mathrm{M})$ or $\mathrm{CH}-3(10 \mu \mathrm{M})$ for $1 \mathrm{~h}$ or $3 \mathrm{~h}$, followed by quantification of transcripts regulated by Nrf2, NFkB, and/or HIF1. In line with the experiments conducted in HEK293 cells, Nrf2activated transcripts, such as Gclm, showed similar regulation with both DMF and CH-3 (Fig. 3(C)). In contrast, Vegf and iNos clearly differed between DMF, CH-3, and controls (Fig. $3(\mathrm{C})$ ). When including an earlier time point, $1 \mathrm{~h}$ following DMF or CH-3 stimulation, this also revealed differences between the two compounds regarding expression in OLs (Fig. S3e). Thus, Gclm, Txn, and $I l 6$ all were significantly different between DMF and $\mathrm{CH}-3$ after $1 \mathrm{~h}$ of stimulation.

When transcriptional patterns at $3 \mathrm{~h}$ were complied into a PCA plot, relatively large differences were recorded between the two cell types (Fig. 3(D)). However, microglia displayed a diverse Nrf2-pattern in response to $\mathrm{CH}-3$ or DMF and thus grouped into two separate clusters away from control stimulation (Fig. 3(D)). In contrast, OLs displayed a more overlapping response to $\mathrm{CH}-3$ and DMF. 
a

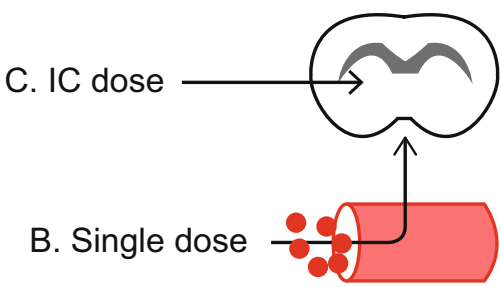

C

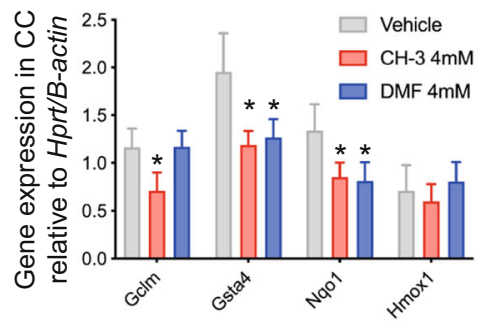

d

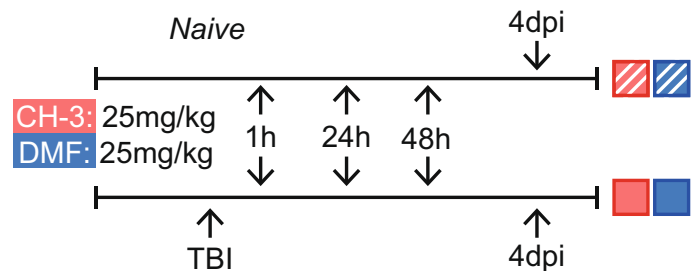

$$
72 \text { Naive }
$$

Oligodendrocytes

h
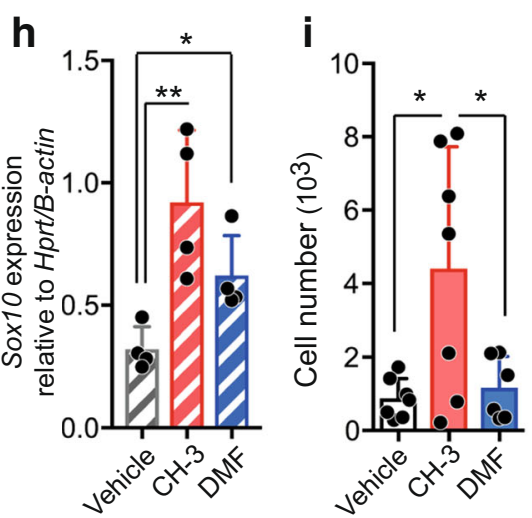

b
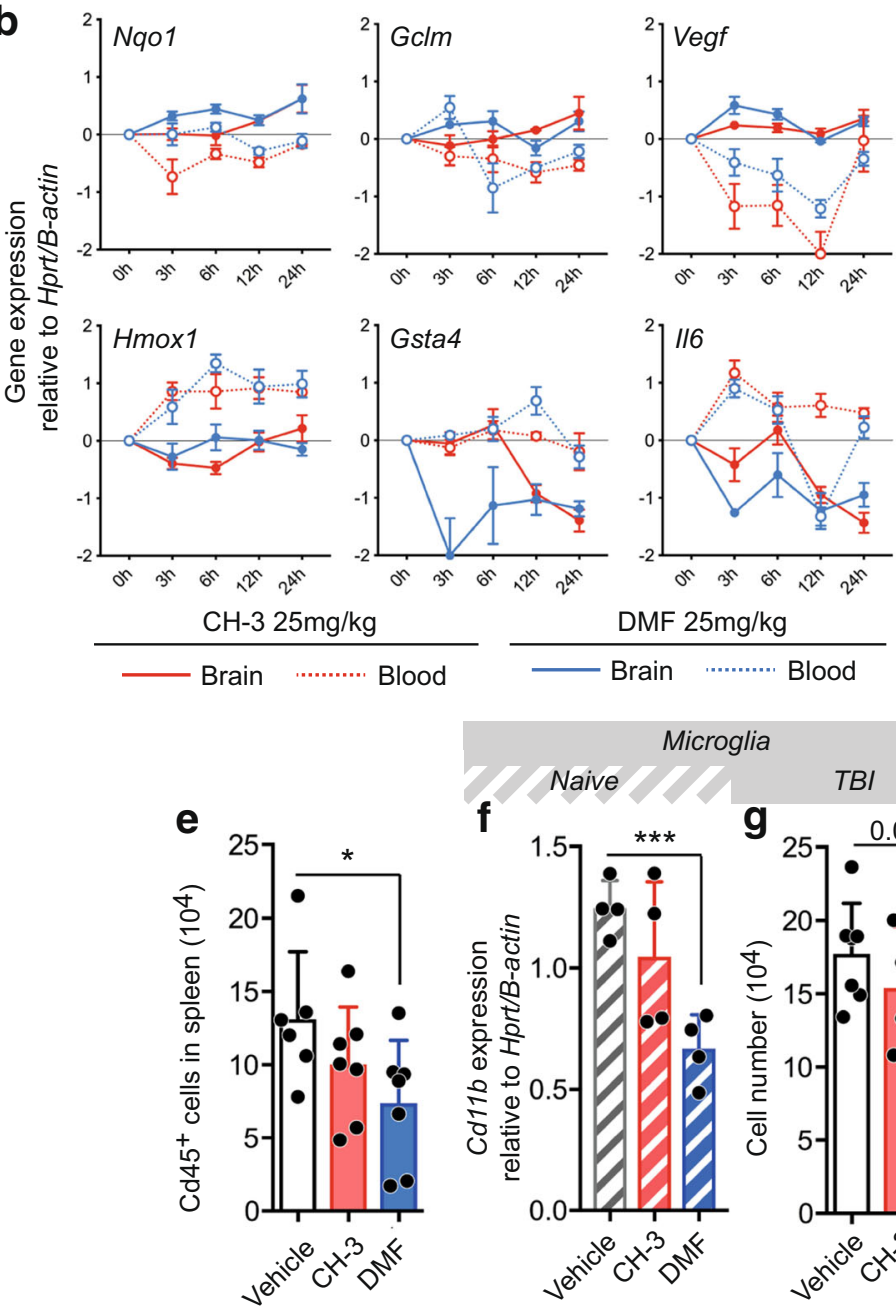
$T B$

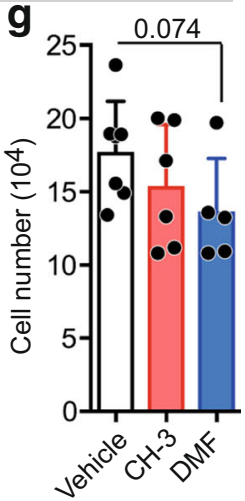

Fig. 4 Naïve in vivo characterization of DMF and CH-3 and following TBI. (A) Experimental outline for routes of administration. (B) Transcriptional pattern in peripheral blood cells (dash) and brain (solid). (C) Transcription pattern in corpus callosum (CC) $5 \mathrm{~h}$ following intra cisterna injections of DMF $(4 \mathrm{mM})$ and $\mathrm{CH}-3(4 \mathrm{mM})(n=5)$. (D) Experimental outline for TBI in combination with intervention. (E) Flow cytometric valuation of $\mathrm{Cd}_{4} 5^{+}$(leukocytes) in the spleen compared to vehicle $(n=6)$. $(\mathrm{F}, \mathrm{G})$ Microglia in brain following $\mathrm{CH}-3$ or DMF treatment assessed by transcription of $C d 11 b(n=4)(\mathrm{F})$, and following
CH-3 or DMF treatment and TBI (g) assessed by flow cytometry $(n=7+$ 5). (H, I) Oligodendrocytes (OL) in brain following $\mathrm{CH}-3$ or DMF treatment assessed by the OL lineage marker $\operatorname{Sox} 10(n=4)(\mathrm{H})$, and following CH-3 or DMF treatment and TBI assessed by flow cytometry $(n=7)(\mathrm{I})$. (J) Levels of NFL in the CSF in sham and following TBI in combination with treatment, detected with ELISA. Error-bars show S.D. Two group comparisons with a control group were done with one-way ANOVA. $* P<0.05, * * P<0.01, * * * P<0.001$ 


\section{CH-3 and DMF Affects Numbers of Pre-OLs and Neurons after TBI}

In RRMS, DMF is administered as an oral tablet preparation. As a first step, we evaluated the capacity of $\mathrm{CH}-3$ and DMF to change brain transcription patterns upon different routes of administration in Dark Agouti (DA) rats (Fig. 4(A)).

To evaluate effects after oral administration, DA rats were given either $\mathrm{CH}-3$ or DMF per body weight or the corresponding volume of vehicle by oral gavage. The dose was decided following titration with increasing doses (Fig. S4a-d). Animals were administered the same dose independent of compound, since similar doses of CH-3 and DMF were effective in primary rat cells (Fig. 3(C, D)). Brain cortex tissue and peripheral blood leukocytes were collected at $3,6,12$, and $24 \mathrm{~h}$ following a single gavage dose. In general, both DMF $(25 \mathrm{mg} / \mathrm{kg})$ and CH-3 $(25 \mathrm{mg} / \mathrm{kg})$ generated larger transcriptional fluctuations in peripheral blood leukocytes compared to brain tissue (Fig. S5). Gclm and Ark1b8, both regulated by $\mathrm{Nrf2}$, were elevated in peripheral blood leukocytes following DMF administration, whereas $\mathrm{CH}-3$ had no discernible effect (Fig. S5). However, Nqo1, also considered regulated by Nrf2, was elevated in brain following $\mathrm{CH}-3$, but not after DMF administration (Fig. 3(B), Fig. S5). In the brain, $\mathrm{CH}-3$ induced elevated transcription of the Nrf2-regulated genes $\mathrm{Nqol}$, Hmoxl, and Gclm. Interestingly, the temporal expression following DMF was largely similar for these transcripts (Fig. 4(B)).

To confirm transcriptional effects of either compounds in the brain parenchyma, $\mathrm{CH}-3$ (4 mM), DMF $(4 \mathrm{mM})$, or the equivalent volume of vehicle was injected in cisterna magna followed by dissection of corpus callosum (CC) $5 \mathrm{~h}$ following the injection. CH-3 and DMF both changed the expression of Nrf2-regulated genes compared to vehicle without discernible differences between the two compounds (Fig. 4(A, C), Fig. S4e).

Nrf2, NFkB, and HIF1 are all activated in responses following TBI and since DMF has been reported to exert beneficial cognitive effects following TBI [4], we repeated that experimental setup to further evaluate $\mathrm{CH}-3$ in direct comparison to DMF (Fig. 4(D)). In brief, rats were administered DMF, CH-3, or corresponding volume of vehicle 1,24 , and $48 \mathrm{~h}$ following TBI. The number of all $\mathrm{Cd} 45^{+}$cells in spleen was significantly lowered following TBI in combination with DMF compared to vehicle (Fig. 4(E)). However, there were no statistical difference in cell numbers between DMF and CH-3.

The reduction of leukocytes in the spleen could potentially be explained by increased migration to the brain after injury, but we could not observe differences across treatments in brain-infiltrating monocytes or total number of $\mathrm{Cd} 45^{+}$cells in the brain (data not shown). In the brain parenchyma, DMF also lowered transcription of the microglia marker
$C d 11 b$ in TBI naïve conditions (administration of drug but no injury) (Fig. 4(F)). Following TBI, DMF suggested a trend for lowered microglia numbers, however not significant (Fig. $4(\mathrm{G}))$. $\mathrm{CH}-3$ displayed a large variance in TBI naïve conditions and showed no significant effects (Fig. 4(F, G)). When assessing differentiated but pre-myelinating OLs, both $\mathrm{CH}-3$ and DMF increased the transcription of the OL-lineage marker Sox10 (Fig. 4(H)). In addition, $\mathrm{CH}-3$ also preserved or increased cell numbers after TBI (Fig. 4(I)). Neurofilament light (NFL) is a component of the functional axon of the nerve cell and is released into cerebrospinal fluid (CSF) upon axonal degeneration, and thus acts as a marker of degree of neuroaxonal degeneration [32]. Animals treated with DMF showed modest, but significantly lower NFL concentrations compared to vehicle, while CH-3 did not differ from control (Fig. 4(J)). NFL levels in sham animals were negligible. In summary, potentially therapeutic effects of DMF or $\mathrm{CH}-3$ appeared to be both tissue and cell-type dependent.

\section{Discussion}

We here addressed the in vitro effects of seven newly synthesized vinyl sulfoximine compounds, and subsequently evaluated the effects on glial cells of the brain in comparison to DMF, currently in clinical use for RRMS. The purpose of this study was to evaluate the transcriptional activity of DMF in different in vitro and in vivo models and to compare this with our de novo synthesized compounds focusing on their effects on brain-resident cells. This is crucial since DMF also engages additional transcription factors next to $\operatorname{Nrf2}[13,15]$.

It is still not unanimously known to what degree beneficial therapeutic effects of DMF can be ascribed its Nrf2-activating effect. Off-target effects of DMF may also contribute. We have previously described the effects of DMF on monocytes and T cells in RRMS, which revealed that DMF exerts prominent oxidizing effects in the systemic compartment, in turn associated with its clinical efficacy [17]. However, DMF has also been shown to have protective effects in experimental disease models, including TBI [4].

Among the newly synthesized vinyl sulfoximine compounds characterized here, $\mathrm{CH}-3$ showed the most promising effect on Nrf2 when evaluated in $p$ TRAF-transfected HEK293 cells. The lowering of NFKB upon addition of DMF to TNF as compared to just TNF underlines the immunosuppressive features described by DMF in mice and man $[33,34]$. The cross comparison of transcripts regulated by DMF and $\mathrm{CH}-3$ revealed only partly overlapping responses. Hence, while Nrf2-regulated transcripts, including GCLM, $N Q O 1$, and GSTA4, showed similar regulation upon exposure to DMF and $\mathrm{CH}-3, V E G F$ and TXNRD1, both regulated by HIF1, were only affected by DMF. These findings suggest that 
CH-3 exerts more limited off-target effects compared to DMF in HEK293 cells.

Upon oral administration, the temporal transcriptional profiles for the Nrf2-regualted genes Nqo1, Hmox1, and Gclm were conserved for both CH-3 and DMF, respectively, where DMF indicated an earlier transcriptional elevation compared to $\mathrm{CH}-3$. The increased transcription was in line with the increase in Nqol and Gclm after DMF and CH-3 in cultured OLs, indicating that OLs may be one of the brain-resident cells responding to $\mathrm{DMF} / \mathrm{CH}-3$. In contrast, microglia during homeostasis in vitro displayed a low degree of changes following either DMF or CH-3. However, this may differ if studied during inflammatory or in vivo conditions. In this study, primary cultures were studied during non-inflammatory conditions to avoid non-compound-mediated NFKB input on Nrf2 activity.

It is also noteworthy that following oral administration, DMF and CH-3 generated temporal Il6 and Vegf transcription that drastically differed in the brain compared to leukocytes in peripheral blood. This was also true for Txn following DMF administration. This finding highlights the need for a more detailed characterization of pharmacodynamics with determination of concentrations in different tissues. The kinetics of these changes also suggested the existence of negative feedback resulting in lowered expression at later time points. Following intrathecal administration to naïve rats, both DMF and $\mathrm{CH}-3$ gave rise to similar expression changes of Nrf2regulated transcripts compared to vehicle in $\mathrm{CC}$ tissue after $5 \mathrm{~h}$. The outcome at this time point is likely to represent an initial drop in Nrf2-regulated gene transcription, as observed for Hmox 1 and $T x n$ at $3 \mathrm{~h}$ following oral administration. Thus, the temporal dynamics in changes in expression patterns likely are affected by differences in in vitro and in vivo conditions.

Nrf2, NFkB, and HIF1, as assessed herein with pTRAF, are all key factors in pathological conditions including TBI [35-37]. Overall, TBI represents a complex inflammatory cascade comprising for example infiltration of immune cells and activation and proliferation of microglia [38], as well as loss of OLs and axonal degeneration [39]. In line with previous publications, DMF lowered the levels of $\mathrm{Cd} 45^{+}$cells, however with a considerable variance $[17,33,34]$.

Furthermore, administration of DMF has also been associated with limited glutathione depletion, suggesting a direct or indirect neuroprotective action in relation to oxidative stress [4]. Our finding that DMF reduces, albeit modestly, NFL after TBI is in line with this notion. We also found that DMF led to a prominent increase in the expression of Gclm and Gsta4 after oral administration, both of which are responsible for intracellular regulation of glutathione. However, the fact that animals treated with $\mathrm{CH}-3$ did not display any clear protective effect on NFL levels in spite of activation of Nrf2 argues that the action of DMF includes additional effects not only depending on activation of Nrf2. On the other hand, we found that $\mathrm{CH}$-3-treated animals displayed a higher number of differentiated but pre-myelinating OLs, which indicates that $\mathrm{CH}$ 3 preserves or stimulates the proliferation of pre-OLs following injury. This suggests that $\mathrm{CH}-3$ contributes to the increase in pre-OLs following injury but $\mathrm{CH}-3$ have limited effect to preserve already myelinating OLs immediately after injury. NFKB is present in pre-OLs but absent in mature OLs [40]. In our in vitro assessment, CH-3 did not affect NFKB, Il6, or IL 8 compared to DMF and this might be a critical aspect why CH-3 animals display a larger population of pre-OLs following TBI compared to DMF-treated animals. Based on this, our data suggests that a limited involvement of NFKB is beneficial for the maintenance or generation of pre-OLs following TBI.

The experiments conducted in primary microglia and OLs cultures extended our observations from the TBI model and clearly highlighted different responses in the two cell types upon exposure to DMF or $\mathrm{CH}-3$. Hence, the response in OLs to CH-3 or DMF was largely similar, while much larger differences were noted in microglia. A possible explanation for this difference is that the transcriptional machinery regulated by the studied transcription factors differ between OLs and microglia. In fact, NFKB has been shown to be low or absent in OLs [40].

\section{Conclusions}

By stepwise assessment of novel vinyl sulfoximine compoiunds and DMF, we demonstrate that one of these, $\mathrm{CH}-3$, display a more Nrf2-specific activating profile compared to DMF. The Nrf2-specific effect is suggested to influence the OL phenotype in terms of promoting proliferation of pre-myelinating cells. In contrast, DMF but not $\mathrm{CH}-3$ reduced concentrations of a nerve cell death biomarker in CSF, suggesting a neuroprotective effect also involve Nrf2-off-target effects. Further studies are needed to extend these observations in order to explore the therapeutic potential of more selective Nrf2 acting compounds in conditions such as demyelination or brain trauma.

Acknowledgments Open access funding provided by Karolinska Institute. We thank Lars Sandberg at the Drug Discovery and Development platform at Science for Life Laboratory for predicting molecular properties.

Author Contributions $\mathrm{KC} / \mathrm{EA} / \mathrm{PA} / \mathrm{FP} / \mathrm{KJ}$ designed the study. $\mathrm{PC} / \mathrm{PA}$ performed compound synthesis. $\mathrm{KC} / \mathrm{KJ} / \mathrm{BE} / \mathrm{EA}$ designed and performed experiments in HEK293 cells. KC/FA/FP designed and performed experiments performed in rats. $\mathrm{KC}$ wrote the manuscript with input from all coauthors.

Funding Information KEC/FP has received funding from NHR Sweden (NN-678-32). ESJA have received funding from Karolinska Institutet, The Swedish Research Council (2017-01872) and The Swedish Cancer Society (2018/333). 


\section{Compliance with Ethical Standards}

Conflict of Interest The authors declare that they have no conflict of interest.

Ethics Approval and Consent to Participate All experiments were approved and performed in accordance with Swedish National Board of Laboratory Animals and the European Community Council Directive (86/609/EEC) under the permits N275-15 and N244-13

Open Access This article is licensed under a Creative Commons Attribution 4.0 International License, which permits use, sharing, adaptation, distribution and reproduction in any medium or format, as long as you give appropriate credit to the original author(s) and the source, provide a link to the Creative Commons licence, and indicate if changes were made. The images or other third party material in this article are included in the article's Creative Commons licence, unless indicated otherwise in a credit line to the material. If material is not included in the article's Creative Commons licence and your intended use is not permitted by statutory regulation or exceeds the permitted use, you will need to obtain permission directly from the copyright holder. To view a copy of this licence, visit http://creativecommons.org/licenses/by/4.0/.

\section{References}

1. Sykiotis GP, Bohmann D. Keap1/Nrf2 signaling regulates oxidative stress tolerance and lifespan in Drosophila. Dev Cell 2008;14(1): 76-85.

2. Brigelius-Flohé R, Flohé L. Basic principles and emerging concepts in the redox control of transcription factors. Antioxid Redox Signal 2011;15(8):2335-81. Mary Ann Liebert, Inc. 140 Huguenot Street, 3rd Floor New Rochelle, NY 10801 USA

3. Copple IM. The Keap1-Nrf2 cell defense pathway-a promising therapeutic target? Adv Pharmacol 2012;63:43-79 Elsevier.

4. Krämer T, Grob T, Menzel L, Hirnet T, Griemert E, Radyushkin K, et al. Dimethyl fumarate treatment after traumatic brain injury prevents depletion of antioxidative brain glutathione and confers neuroprotection. J Neurochem 2017;143(5):523-33. Wiley/Blackwell (10.1111)

5. Ellrichmann G, Petrasch-Parwez E, Lee D-H, Reick C, Arning L, Saft C, et al. Efficacy of fumaric acid esters in the R6/2 and YAC128 models of Huntington's disease. PLoS ONE 2011;6(1): e16172. Kleinschnitz C, editor.

6. Moharregh-Khiabani D, Blank A, Skripuletz T, Miller E, Kotsiari A, Gudi V, et al. Effects of fumaric acids on cuprizone induced central nervous system de- and remyelination in the mouse. PLoS ONE 2010;5(7):e11769.s. Kleinschnitz C, editor

7. van der Veen RC, Dietlin TA, Hofman FM, Pen L, Segal BH, Holland SM. Superoxide prevents nitric oxide-mediated suppression of helper T lymphocytes: decreased autoimmune encephalomyelitis in nicotinamide adenine dinucleotide phosphate oxidase knockout mice. J Immunol 2000;164(10):5177-83.

8. Noubade R, Wong K, Ota N, Rutz S, Eidenschenk C, Valdez PA, et al. NRROS negatively regulates reactive oxygen species during host defence and autoimmunity. Nature 2014;509(7499):235-9. Nature Publishing Group

9. Kobayashi EH, Suzuki T, Funayama R, Nagashima T, Hayashi M, Sekine H, et al. Nrf2 suppresses macrophage inflammatory response by blocking proinflammatory cytokine transcription. Nat Commun 2016;7:11624.

10. Fox RJ, Miller DH, Phillips JT, Hutchinson M, Havrdova E, Kita $\mathrm{M}$, et al. Placebo-controlled phase 3 study of oral BG-12 or glatiramer in multiple sclerosis. N Engl J Med 2012;367(12): 1087-97. Massachusetts Medical Society

11. Gold R, Kappos L, Arnold DL, Bar-Or A, Giovannoni G, Selmaj K, et al. Placebo-controlled phase 3 study of oral BG-12 for relapsing multiple sclerosis. N Engl J Med 2012;367(12):1098-107. Massachusetts Medical Society

12. Linker RA, Lee D-H, Ryan S, van Dam AM, Conrad R, Bista P, et al. Fumaric acid esters exert neuroprotective effects in neuroinflammation via activation of the Nrf2 antioxidant pathway. Brain 2011;134(Pt 3):678-92.

13. Schulze-Topphoff U, Varrin-Doyer M, Pekarek K, Spencer CM, Shetty A, Sagan SA, et al. Dimethyl fumarate treatment induces adaptive and innate immune modulation independent of Nrf2. Proc Natl Acad Sci U S A 2016;113(17):4777-82.

14. Johansson K, Cebula M, Rengby O, Dreij K, Carlström KE, Sigmundsson K, et al. Cross Talk in HEK293 Cells Between Nrf2, HIF, and NF-kB Activities upon Challenges with Redox Therapeutics Characterized with Single-Cell Resolution. Antioxid Redox Signal 2017;26(6):229-46. Mary Ann Liebert, Inc. 140 Huguenot Street, 3rd Floor New Rochelle, NY 10801 USA

15. Kornberg MD, Bhargava P, Kim PM, Putluri V, Snowman AM, Putluri N, et al. Dimethyl fumarate targets GAPDH and aerobic glycolysis to modulate immunity. Science 2018;360(6387):449-53.

16. Chen H, Assmann JC, Krenz A, Rahman M, Grimm M, Karsten $\mathrm{CM}$, et al. Hydroxycarboxylic acid receptor 2 mediates dimethyl fumarate's protective effect in EAE. J Clin Invest 2014;124(5): 2188-92. American Society for Clinical Investigation

17. Carlström KE, Ewing E, Granqvist M, Gyllenberg A, Aeinehband S, Enoksson SL, et al. Therapeutic efficacy of dimethyl fumarate in relapsing-remitting multiple sclerosis associates with ROS pathway in monocytes. Nat Commun 2019;10(1):3081.

18. Penner N, Sejbaek T, Woodward C, Edwards K, Mendoza J, Illes Z. Cerebrospinal Fluid Penetration of Dimethyl Fumarate in Patients With Multiple Sclerosis. th Congress of the European Committee for Treatment Research in Multiple Sclerosis ECTRIMS 2018:1-1.

19. Woo SY, Kim JH, Moon MK, Han SH, Yeon SK, Choi JW, et al. Discovery of vinyl sulfones as a novel class of neuroprotective agents toward Parkinson's disease therapy. J Med Chem 2014;57(4):1473-87.

20. Lee JA, Kim JH, Woo SY, Son HJ, Han SH, Jang BK, et al. A novel compound VSC2 has anti-inflammatory and antioxidant properties in microglia and in Parkinson's disease animal model. Br J Pharmacol 2015;172(4):1087-100. Wiley/Blackwell (10.1111)

21. Mitsuishi Y, Motohashi H, Yamamoto M. The Keap1-Nrf2 system in cancers: stress response and anabolic metabolism. Front Oncol. Frontiers 2012;2:200.

22. Abbas K, Breton J, Planson A-G, Bouton C, Bignon J, Seguin C, et al. Nitric oxide activates an Nrf2/sulfiredoxin antioxidant pathway in macrophages. Free Radic Biol Med 2011;51(1):107-14.

23. Nimer Al F, Beyeen AD, Lindblom R, Ström M, Aeinehband S, Lidman O, et al. Both MHC and non-MHC genes regulate inflammation and T-cell response after traumatic brain injury. Brain Behav Immun 2011;25(5):981-90.

24. Nimer Al F, Ström M, Lindblom R, Aeinehband S, Bellander B-M, Nyengaard JR, et al. Naturally occurring variation in the Glutathione-S-Transferase 4 gene determines neurodegeneration after traumatic brain injury. Antioxid Redox Signal 2013;18(7):78494. Mary Ann Liebert, Inc. 140 Huguenot Street, 3rd Floor New Rochelle, NY 10801 USA

25. Goldberg FW, Kettle JG, Kogej T, Perry MWD, Tomkinson NP. Designing novel building blocks is an overlooked strategy to improve compound quality. Drug Discov Today 2015;20(1):11-7.

26. Boger DL. The Difference a Single Atom Can Make: Synthesis and Design at the Chemistry-Biology Interface. J Org Chem 2017;82(23):11961-80. American Chemical Society 
27. Chinthakindi PK, Nandi GC, Govender T, Kruger HG, Naicker T, Arvidsson PI. An efficient protecting group free synthesis of vinvylic sulfoximines via Horner-Wadsworth-Emmons reaction. Synlett 2016; 27(09):1423-27. Thieme

28. Lemasson FA, Strunk T, Gerstel P, Hennrich F, Lebedkin S, BarnerKowollik C, et al. Selective dispersion of single-walled carbon nanotubes with specific chiral indices by poly(N-decyl-2,7-carbazole). J Am Chem Soc 2011;133(4):652-5. American Chemical Society

29. Teng F, Yu J-T, Jiang Y, Yang H, Cheng J. A copper-mediated oxidative N-cyanation reaction. Chem Commun (Camb) 2014;50(61):8412-5. The Royal Society of Chemistry

30. Gupta S, Chaudhary P, Muniyappan N, Sabiah S, Kandasamy J. Copper promoted $\mathrm{N}$-alkylation of sulfoximines with alkylboronic acid under mild conditions. Org Biomol Chem 2017;15(40):84938. The Royal Society of Chemistry

31. Scannevin RH, Chollate S, Jung M-Y, Shackett M, Patel H, Bista P, et al. Fumarates promote cytoprotection of central nervous system cells against oxidative stress via the nuclear factor (erythroid-derived 2)-like 2 pathway. J Pharmacol Exp Ther 2012;341(1):27484.

32. Norgren N, Rosengren L, Stigbrand T. Elevated neurofilament levels in neurological diseases. Brain Res 2003;987(1):25-31.

33. Wu Q, Wang Q, Mao G, Dowling CA, Lundy SK, Mao-Draayer Y. Dimethyl Fumarate Selectively Reduces Memory T Cells and Shifts the Balance between Th1/Th17 and Th2 in Multiple Sclerosis Patients. J Immunol 2017;198(8):3069-80. American Association of Immunologists

34. Gross CC, Schulte-Mecklenbeck A, Klinsing S, Posevitz-Fejfár A, Wiendl H, Klotz L. Dimethyl fumarate treatment alters circulating T helper cell subsets in multiple sclerosis. Neurol Neuroimmunol Neuroinflamm 2016;3(1):e183. Wolters Kluwer Health, Inc. on behalf of the American Academy of Neurology
35. Li L, Tan J, Miao Y, Lei P, Zhang Q. ROS and Autophagy: Interactions and Molecular Regulatory Mechanisms. Cell Mol Neurobiol 2015;35(5):615-21. Springer US

36. Zhang M, An C, Gao Y, Leak RK, Chen J, Zhang F. Emerging roles of Nrf2 and phase II antioxidant enzymes in neuroprotection. Prog Neurobiol 2013;100:30-47.

37. Sandberg M, Patil J, D'Angelo B, Weber SG, Mallard C. NRF2regulation in brain health and disease: implication of cerebral inflammation. Neuropharmacology 2014;79:298-306.

38. Donat CK, Scott G, Gentleman SM, Sastre M. Microglial Activation in Traumatic Brain Injury. Front Aging Neurosci 2017;9:208. Frontiers

39. Flygt J, Djupsjö A, Lenne F, Marklund N. Myelin loss and oligodendrocyte pathology in white matter tracts following traumatic brain injury in the rat. Eur J Neurosci 2013;38(1):2153-65. John Wiley \& Sons, Ltd (10.1111)

40. Blank T, Prinz M. NF-KB signaling regulates myelination in the CNS. Front Mol Neurosci 2014;7(92):47. Frontiers

41. Mireille M.J.P.E. Sthijns, Paul M. Schiffers, Ger M. Janssen, Kristien J.A. Lemmens, Bart Ides, Philippe Vangrieken, Freek G. Bouwman, Edwin C. Mariman, Irina Pader, Elias S.J. Arnér, Katarina Johansson, Aalt Bast, Guido R.M.M. Haenen, (2017) Rutin protects against $\mathrm{H} 2 \mathrm{O} 2$-triggered impaired relaxation of placental arterioles and induces Nrf2-mediated adaptation in Human Umbilical Vein Endothelial Cells exposed to oxidative stress. Biochimica et Biophysica Acta (BBA) - General Subjects 1861 (5):1177-1189

Publisher's Note Springer Nature remains neutral with regard to jurisdictional claims in published maps and institutional affiliations. 